\title{
Conducting polymers grown in hydrogel scaffolds coated on neural prosthetic devices
}

\author{
Dong-Hwan Kim, ${ }^{1}$ Mohammad Abidian, ${ }^{1}$ David C. Martin ${ }^{1,2,3}$ \\ ${ }^{1}$ Biomedical Engineering, The University of Michigan, Ann Arbor, Michigan 48109 \\ ${ }^{2}$ Materials Science and Engineering, The University of Michigan, Ann Arbor, Michigan 48109 \\ ${ }^{3}$ Macromolecular Science and Engineering Center, The University of Michigan, Ann Arbor, Michigan 48109
}

Received 3 October 2003; revised 24 May 2004; accepted 1 June 2004

Published online 27 October 2004 in Wiley InterScience (www.interscience.wiley.com). DOI: 10.1002/jbm.a.30124

\begin{abstract}
The conducting polymer polypyrrole (PPy) was electrochemically grown on hydrogel scaffolds deposited on the surface of microfabricated neural prosthetic devices. It is shown that the pyrrole monomer can be grown vertically through the hydrogel layer up to the surface without affecting the adjacent sites on the probes. The electrochemical properties of the conducting polymer-modified hydrogels were studied by impedance spectroscopy and cyclic voltammetry. It is also found that the conducting polymers could still be readily grown through the hydrogel after the micro-
\end{abstract}

structure is disrupted by freeze drying. Impedance measurements at the biologically important frequency of $1 \mathrm{kHz}$ showed that the minimum impedance of this polymer-modified hydrogel was $7 \mathrm{k} \Omega$. This is much lower than the minimum impedance of polypyrrole film $(\sim 100 \mathrm{k} \Omega)$. (C) 2004 Wiley Periodicals, Inc. J Biomed Mater Res 71A: 577-585, 2004

Key words: hydrogel; conducting polymer; polypyrrole; neural microelectrode; neural recording

\section{INTRODUCTION}

Microfabricated neural prosthetic devices for the central or peripheral nervous system have evolved rapidly. ${ }^{1}$ Details of the interfacial contact between the electrode and the brain tissue are important not only for promoting cell adhesion but also for facilitating charge transport. ${ }^{1,2}$ Microelectrodes have proven to be inadequate for long-term implantation, with cellular encapsulation playing a significant role in limiting neural communication. ${ }^{3,4}$ Although the precise origin of implant failure is not yet known, recent efforts have shown improvements in performance. Some researchers advocate that device failures are related to isolation of the electrode sites, which is mainly caused by scar formation after implantation; others consider the trauma to neurons caused from micromotion due to the mechanical differences between the electrode and brain tissue. ${ }^{5}$ Fibrous tissue encapsulation is the final stage of wound-healing response to implanted foreign materials. Generally, it consists of an inner layer of macrophages, a concentric fibrous tissue, and an outer vascularized tissue layer. ${ }^{6}$ Presumably the performance of these devices could be improved by promot-

Correspondence to: D. C. Martin; e-mail milty@umich.edu ing better integration within the nervous system and minimizing the host response. ${ }^{3,7}$

Hydrogels are widely used biomaterials that have found many pharmaceutical and biomedical applications, including scaffolds for tissue engineering, wound dressings, and delivery carriers for bioactive reagents. Hydrogels are generally found to be biocompatible due to their high water content and low interfacial tension with the surrounding biological environment. ${ }^{8}$ The mechanical properties of hydrogels can be tailored to be similar to brain tissue $(\sim 10 \mathrm{kPa}){ }^{9} \mathrm{Hy}-$ drogel coatings should provide an additional mechanical buffer layer between the hard silicon-based probe and the soft brain tissue. In addition, the reswelling of dried gels following implantation should better anchor the position of the probe in the tissue. Another advantage is that various bioactive reagents such as growth factors or anti-inflammatory drugs can be embedded in the hydrogel coatings. Hydrogels can undergo volume changes in response to external electric stimuli. However, these electrical stimuli are not rapidly transmitted through the hydrogel network because of the low electronic conductivity of the polymer matrix. ${ }^{10}$

Conducting electroactive polymers including polypyrrole, polyaniline, and polythiophene have been used to modify biosensors and bioelectronics. ${ }^{11,12} \mathrm{Be}-$ cause the physiological environment involves pro- 
cesses at the interface between electrode and electrolyte, there has been considerable experimental and theoretical attention devoted to increasing the surface area of the electrodes. ${ }^{13-15}$ Although the conducting polymer allows itself to be in contact with the electrolyte at the molecular level, the intrinsically hydrophobic character of most conducting polymers requires that these processes happen at the surface of the electrode. Surface modification by conducting polymers is intended to improve probe sensitivity, suppress the effect of interfering reactions, and encourage desired tissue responses such as angiogenesis. ${ }^{16}$ Of these conducting polymers, polypyrrole has been widely studied because of the aqueous solubility of the monomer, low oxidation potential, high conductivity, and compatibility with mammalian cells. ${ }^{17}$ PSS (Polystyrene sulfonate) was chosen as the dopant material because of its stability and biocompatibility. ${ }^{17}$ When a polyelectrolyte with a negative charge serves as the counter ion, the polymerization process can be represented as follows:

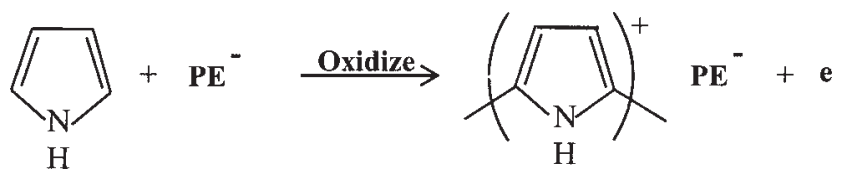

where $\mathrm{PE}^{-}$stands for polyelectrolyte. Based on this mechanism, bioactive surfaces have been created by incorporating species such as heparin, poly(hyaluronic acid), nerve growth factors, and red blood cells. ${ }^{18-23}$ In vitro studies involving biologic responses of polypyrrole are already taking place. ${ }^{24}$ Recent investigations involve the use of conducting polymers such as polypyrrole and poly(3,4-ethylenedioxythiophene) (PEDOT); and incorporating bioactive molecules such as nerve growth factor (NGF) to enhance the neural attraction to the electrode.

The electrodes on the surface of the probe are actively involved in the stimulation and recording of impulses from neurons. The impedance of polymercoated electrodes has been found to be strongly correlated to the morphology of the coating. ${ }^{16,25}$

Kim, Spinks, and Wallace showed that conducting polymers could be electrochemically synthesized inside a hydrogel support matrix. ${ }^{31}$ This article shows that polypyrrole can be grown vertically through the hydrogel layer up to the surface without affecting the adjacent sites on the neural probes. This conducting polymer in the hydrogel layer has an extremely large surface area. This significantly reduces the impedance of the electrode and promotes efficient signal transport with the neurons. However, to allow neovascularization in the hydrogel layer and the neurons to grow into the hydrogel layers, the pore size of the usual alginate hydrogels may not be sufficient. Therefore, lyophilized hydrogels were also investigated.
These have a much larger pore size than the unmodified hydrogels as a scaffold for polymerization of the conducting polymers.

The hydrogels were deposited by a dipping method, with the use of a $1 \mathrm{wt} \%$ alginate solution that was gelled by ionic crosslinking with $0.5 \mathrm{M} \mathrm{CaCl}_{2}$. After the hydrogel was coated along the shank of probes, PPy/PSS was grown galvanostatically on the gold electrode of the probes at a current density of 4.8 $\mathrm{mA} / \mathrm{cm}^{2}$. Growth times were restricted to less than $1 \mathrm{~h}$ because the electric forces deformed the hydrogel severely in the monomer solution after approximately $1 \mathrm{~h}$ of polymerization. To characterize the polymerfunctionalized electrodes, their impedance and cyclic voltammetry were compared with those of planar gold electrodes with the same geometric surface area by electrochemical impedance spectroscopy (EIS).

\section{EXPERIMENT}

\section{Materials}

The sodium alginates were purchased form ProNova Biomedical (Norway). MVG alginate, a high G-containing alginate (M:G ratio 40:60 as specified by the manufacturer) was used in this study. The pyrrole monomer and PSS were purchased from Aldrich and used without any prior purification. All other chemicals were purchased form Sigma (St. Louis, MO) unless otherwise stated. Solutions of sodium alginate at a concentration of $1 \mathrm{wt} \%$ were freshly prepared by dissolving the alginate powder in double-distilled water, while mixing with a magnetic stirrer. $\mathrm{CaCl}_{2} 0.5 \mathrm{M}$ was used as a source of calcium ions to initiate gelation.

\section{Material preparation}

Microfabricated silicon probes were provided by the Center for Neural Communication Technology at the University of Michigan. The microfabricated silicon substrate supports an array of thin-film conductors that are insulated above and below by deposited dielectrics of silicon dioxide and silicon nitride. Openings in the upper dielectrics along the probe define vertical connections to stimulating or recording sites that are sputtered with gold or iridium on the top dielectrics for interfacing with the tissue. At the rear of the probe, gold bond pads are present to connect with off-chip instrumentation.

The hydrogels were deposited by a dipping method, with the use of a $1 \mathrm{wt} \%$ alginate solution that was gelled by ionic crosslinking with $0.5 \mathrm{M} \mathrm{CaCl}$. After coating the hydrogel along the shank of probes, PPy/PSS was grown galvanostatically on the gold or iridium electrode sites $\left(1250 \mu \mathrm{m}^{2}\right.$ in area) of the neural probes. The electrical supply was a potentiostat/galvanostat EG\&G Model 283. The reaction was performed in a three-electrode cell. A platinum foil was used 


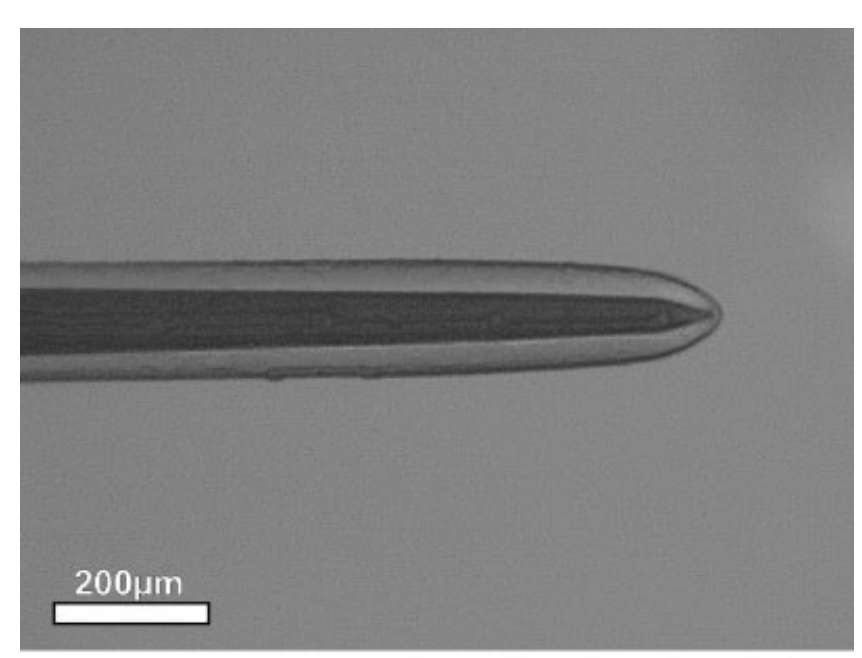

(a)

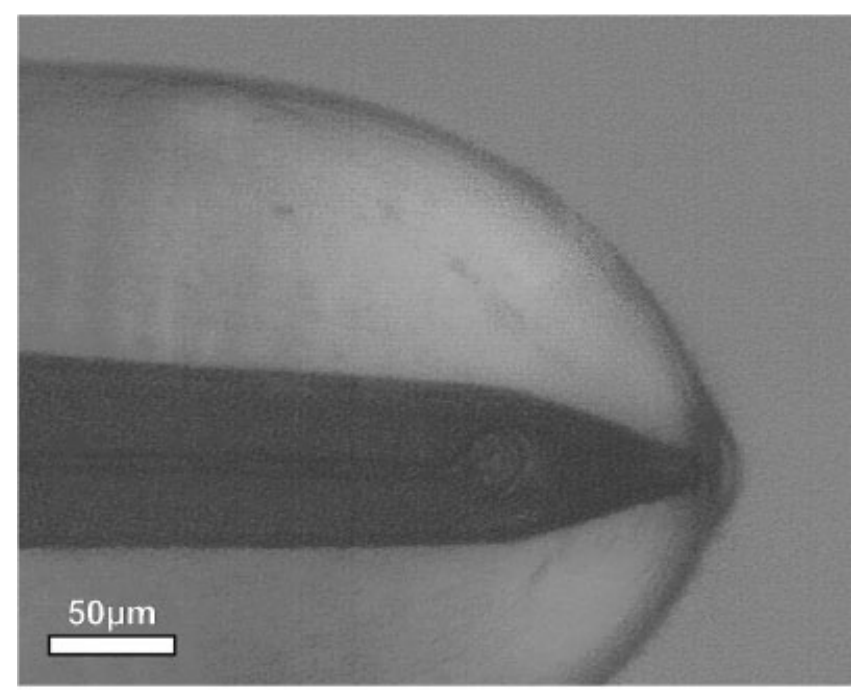

(b)

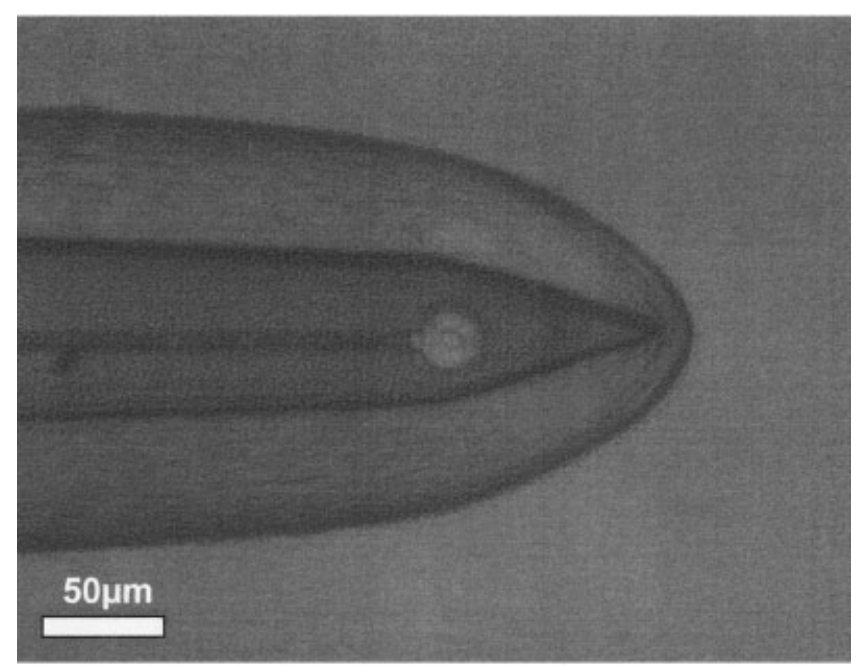

(c) as the counter electrode and a saturated calomel electrode (SCE) was used as the reference electrode. A site on the probe was used as the working electrode. The monomer solution (40 $\mathrm{mL}$ ) containing $0.2 \mathrm{M}$ pyrrole monomer, $0.2 \mathrm{M}$ PSS, and $4 \mathrm{~mL}$ of $1 M \mathrm{CaCl}_{2}$ was purged with $\mathrm{N}_{2}$ for approximately $10 \mathrm{~min}$ to prevent oxidation of the monomer before use. In the galvanostatic mode, a current density of $4.8 \mathrm{~mA} / \mathrm{cm}^{2}$ was used. The amount of material deposited onto the electrode site was controlled by changing the deposition time.

\section{Lyophilizing the hydrogel}

The detailed lyophilizing procedures are described elsewhere. ${ }^{27}$ Briefly, sodium alginate dissolved in double-distilled water was cross-linked on the electrode with calcium ions. Freezing of the gels was accomplished by either placing the hydrogel in a $-20^{\circ} \mathrm{C}$ freezer, or by submerging them into liquid nitrogen $\left(-196^{\circ} \mathrm{C}\right)$ immediately after gelation to prevent drying of the gel. The frozen plates were transferred to vacuum vessels and lyophilized at $<10$ mbar until dry.

\section{Impedance spectroscopy measurements}

An EG\&G electrochemical impedance system was used to obtain impedance spectra of the electrode sites. The apparatus was comprised of an EG\&G Model 283 potentiostat/ galvanostat and an EG\&G Model 1025 frequency response analyzer. The system was operated under computer control with the use of an EIS software package from EG\&G. A solution of $0.1 \mathrm{M}$ phosphate buffer solution $(\mathrm{pH}=7)$ was used as the electrolyte in a three-electrode cell. The electrode setup was the same as that used in the electrochemical deposition. An ac sinusoidal signal of 5-mV amplitude was used, and the dc potential was set to $0 \mathrm{~V}$. The value of the impedance was determined over a range of 10-100,000 Hz.

\section{Cyclic voltammetry}

Cyclic voltammetry (CV) was performed with the use of a house-designed potentiostat, combined with a phaselock amplifier (Stanford Research System SR 830) and multiplexer (National Instruments SC-2062). The threeelectrode cell setup was the same as that used for impedance spectroscopy. A scan rate of $100 \mathrm{mV} / \mathrm{s}$ was used and the potential on the working electrode was swept between -0.9 and $0.5 \mathrm{~V}$ versus SCE. These limits were wide enough to include the reversible redox reaction and nar-

Figure 1. Optical microscope images of the hydrogel coatings on the probes by dipping method. (a) Overall view of the hydrogel coatings at low magnification, $(b, c)$ closer views of coatings with various thicknesses $[\sim 100 \mu \mathrm{m}$ for $(b)$, $\sim 50 \mu \mathrm{m}$ for (c)]. 


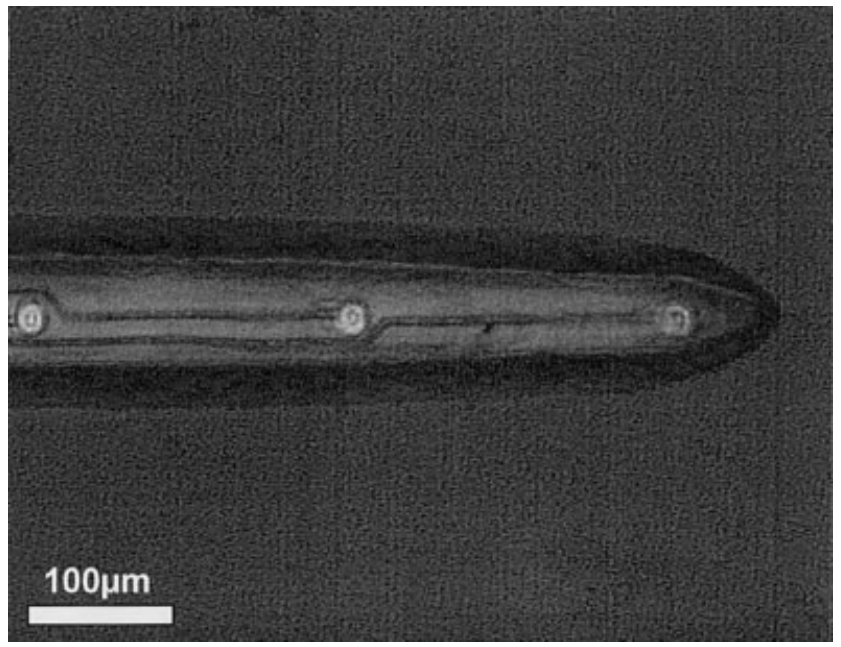

Figure 2. The dehydrated hydrogel coating on the probe in ambient air.

row enough to avoid overoxidation and remain within the water window. Before each $\mathrm{CV}$ was recorded, several cycles were swept to ensure that the film reached its stable state.

\section{Acute neural recording}

Acute experiments in guinea pig were used to verify and characterize in vivo electrical functionality of the hydrogel itself. NIH guidelines for the care and use of laboratory animals (NIH Publication No. 85-23 Rev. 1085) have been observed. The detailed procedures are given elsewhere. ${ }^{16}$ The guinea pig was anesthetized with ketamine and xylazine. The animal was then placed on a DC-powered heating pad to prevent a drop in core body temperature. The skull was fixed with a high degree of stability to a rigid bar after incision on the head of $1.5 \mathrm{in}$. A craniotomy was performed to expose the dorsal surface of the cerebellum, the dura was removed, and the probe was inserted. The brain was then covered with a warm agar solution to prevent desiccation and reduce brain pulsation. The hydrogel-coated probes with various thicknesses from 15 to $50 \mu \mathrm{m}$ were advanced until a neuron was recorded at every single site. Neural data were recorded and analyzed to find the neural spikes for each channel with various thicknesses of hydrogels.

\section{RESULTS AND DISCUSSION}

\section{Hydrogel coating on the surface of the probes}

Uniformly coated hydrogel layers with various thicknesses have been successfully produced along the shank of micromachined probes to provide an additional mechanical buffer layer and anchor the po- sition of the probe in the tissue. The optical micrograph shown in Figure 1 demonstrates the hydrogel layer on a silicon probe. Figure 1(a) shows the overall view of the hydrogel coatings at low magnification, and Figure $1(b, c)$ shows closer views of coatings with various thicknesses. In this study, the thickness of the hydrogels was kept at less than $100 \mu \mathrm{m}$ to prevent ingrowing neurons from possible oxygen deprivation. ${ }^{8}$ Figure 2 shows the dehydrated hydrogel coating on the probe. The dip-coated hydrogel dried quickly in air and shrunk in volume. The hydrogels can be readily returned to nearly their original shape by reabsorbing water. ${ }^{28}$

The thickness of the hydrogel layer was controlled by changing the number of dips of the probes into alginate solution as well as the concentration of the alginate in the solution. A uniform coating was relatively easy to obtain by using a low concentration of alginate solution. As the dipping proceeded, the hydrogel layers grew thicker ( $\sim 50 \mu \mathrm{m} / 20$ dips).

The electronic and electrochemical properties of the unmodified hydrogel were measured with the use of electrochemical impedance spectroscopy (EIS) and cyclic voltammetry $(\mathrm{CV}) .{ }^{16,25}$ Figure 3 shows the impedance spectra of hydrogel coatings on the electrode compared with the gold electrode spectrum as a control. After being coated with hydrogel $100 \mu \mathrm{m}$ thick or less, the impedance was slightly higher than the baregold electrode over the $1-1-\mathrm{kHz}$ range and similar to the bare gold beyond $1 \mathrm{kHz}$. Because the reswollen hydrogel absorbed water and a variety of ions from the buffer solution, this result is consistent with expected high conductivity of the ion-laden water.

To ensure that the hydrogel coating along the shank of the probe did not affect neural recording and stim-

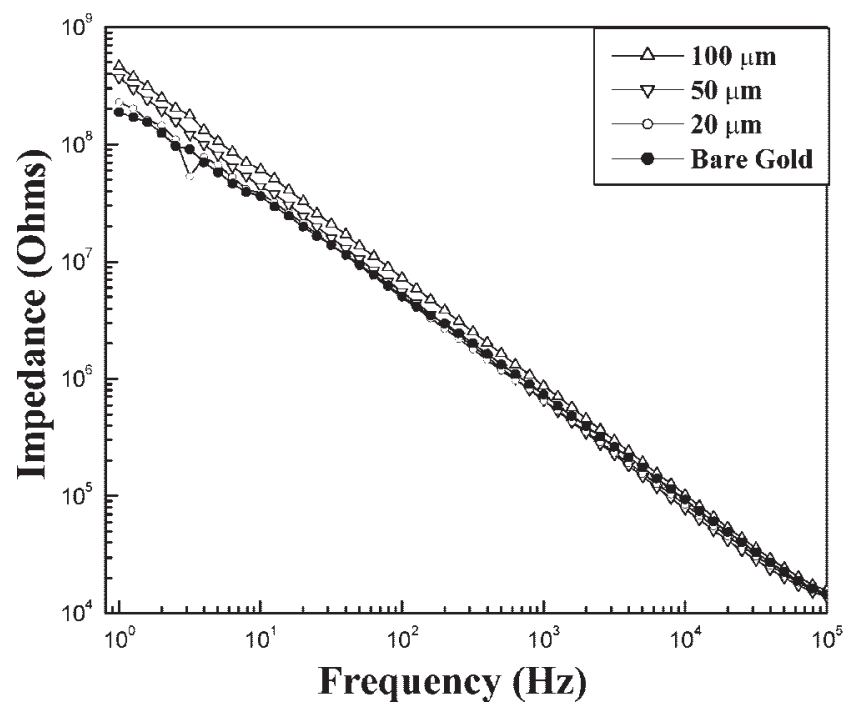

Figure 3. Impedance spectra of hydrogel coatings with various coating thicknesses on the electrode compared with the gold electrode spectrum as a control. 


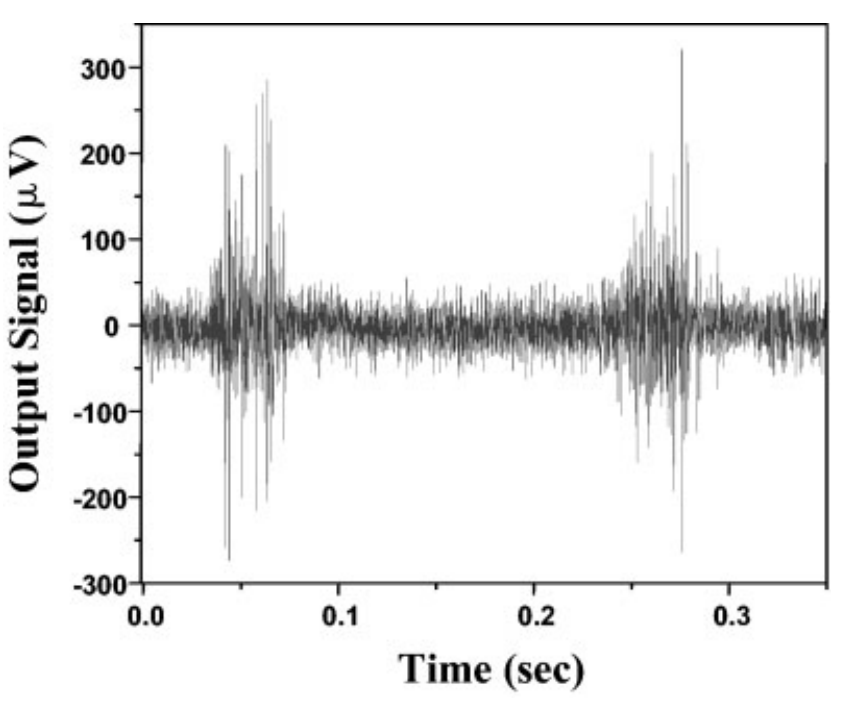

Figure 4. Acute recording data from the cerebellum of a guinea pig.

ulating properties, in vivo recording tests were performed at the Kresge Hearing Research Center. The acute recording data from the cerebellum of guinea pigs showed that every single electrode of the hydrogel-coated probe could transport charge as efficiently as the uncoated probes (Figure 4).

\section{Polymerization of the PPy/PSS in the hydrogel coatings}

It was found that the pyrrole monomer can be grown vertically from the site to the surface of the hydrogel layer without affecting the adjacent sites. Figure 5(a) shows the optical microscope images of the extent of conducting polymer growth in the hydrogel coating. The top view of the conducting polymer shows a cloud-like morphology instead of the dense film structure usually observed. ${ }^{16,25}$ The film was blackish in color, which is characteristic of the oxidized state of polypyrrole. The optical microscope images show that the conducting polymer is not placed just on the electrode. The lateral view reveals the three-dimensional cloudlike morphology of the conducting polymer within the hydrogel directly above the electrode. Because new polymer formation can presumably only be initiated electrochemically at a conducting surface, these results suggest that the entire polypyrrole phase is interconnected. ${ }^{16,25}$ The detailed morphology of this system is currently being investigated with the use of SEM and TEM.

In contrast to the conducting polymer films that are deposited directly on the electrode, this conducting polymer deposited in the hydrogel layer evidently has an extremely large amount of surface area, as shown schematically in Figure 6. This reduces the impedance of the electrode and should promote efficient signal transport with neurons. These hydrogel electrodes coated with conducting polymers are interesting for sensor applications because of the larger surface area over which electrolytes can access the electrode. Figure 7 shows the change in impedance as a function of polymerization time, and as a function of dehydration. After 20 min of polymerization of the polypyrrole, the impedance was significantly decreased over the frequency range from 10 to $100,000 \mathrm{~Hz}^{26}$ compared to the bare gold electrode with the same geometric surface area. The impedance of this polymer at the biologically important frequency of $1 \mathrm{kHz}$ was $120 \mathrm{k} \Omega$. Additionally the impedance of the conducting polymermodified hydrogel was much lower than the impedance of intact hydrogel coatings. This means the conducting polymer-modified hydrogel can compen-

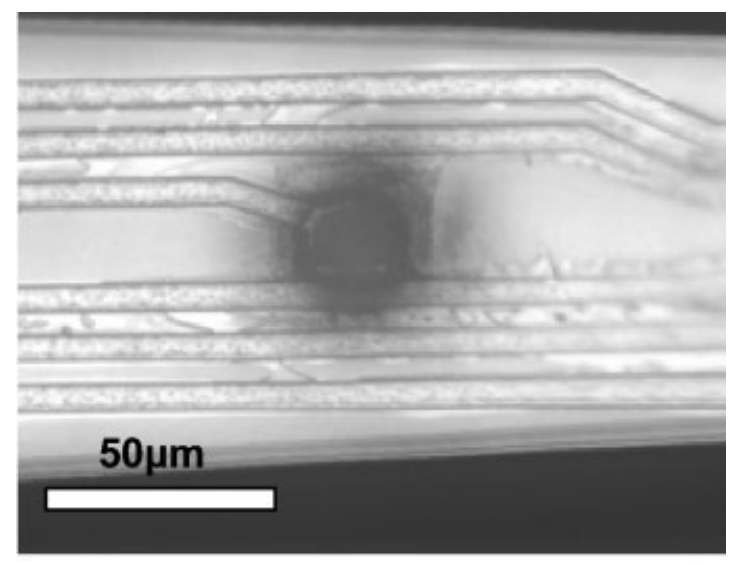

(a)

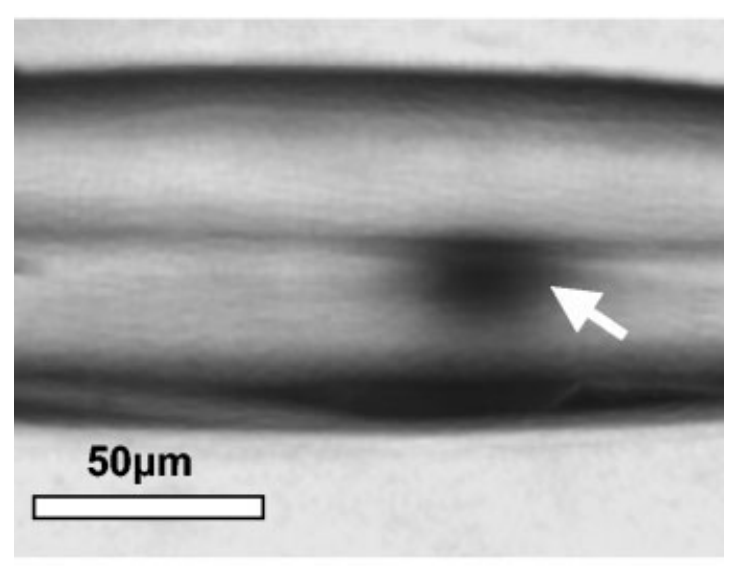

(b)

Figure 5. Optical microscope images of the extent of conducting polymer growth in the hydrogel coating. (a) Top view of the conducting polymer, and (b) lateral view revealing the three-dimensional cloud-like morphology (white arrow) of the conducting polymer within the hydrogel directly above the electrode. 


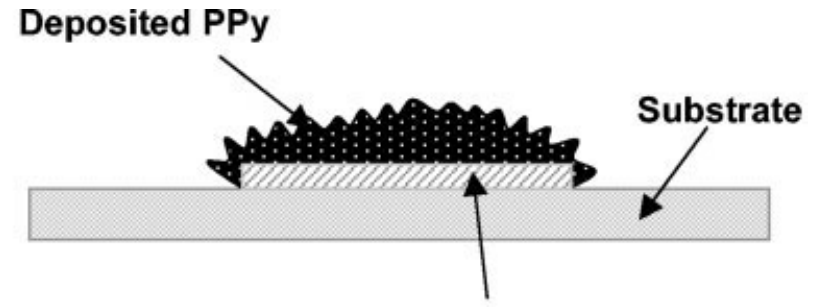

Gold electrode

(a)

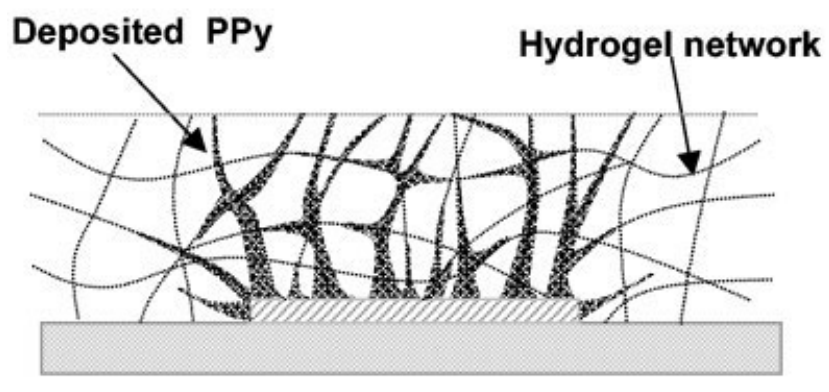

(b)

Figure 6. Schematic of the film-like conducting polymer on the gold electrode (a) and the cloud-like conducting polymer on the gold electrode polymerized through the hydrogel matrix (b).

sate for the slight increase in impedance due to the intact hydrogel coatings. This will presumably allow us to modify the surface of the probes with little or no change in impedance by embedding various growth

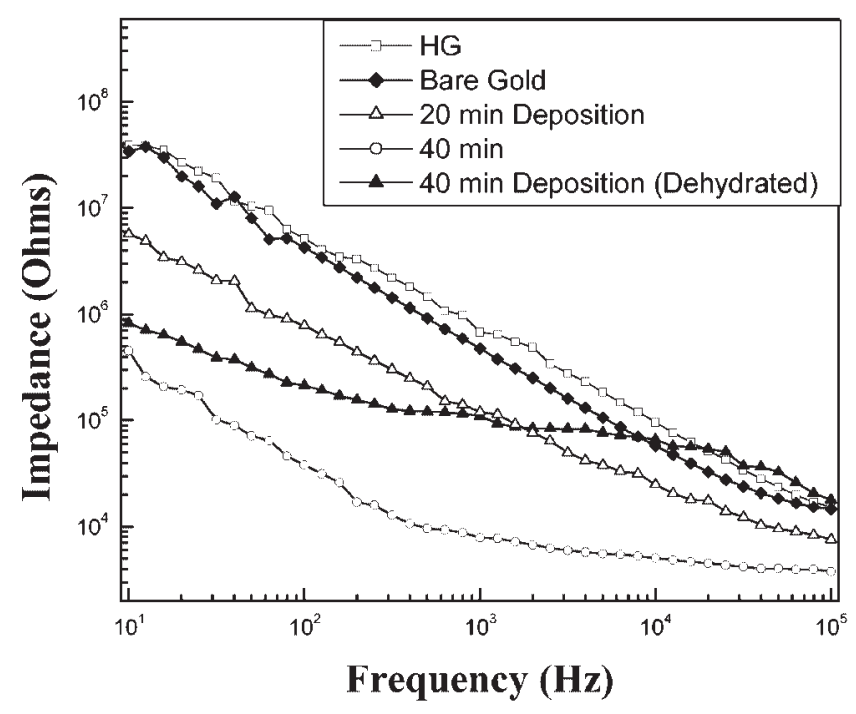

Figure 7. Impedance spectra as a function of polymerization time, and as a function of dehydration. The impedance of the conducting polymer-modified hydrogel was much lower than the impedance of intact hydrogel coatings.

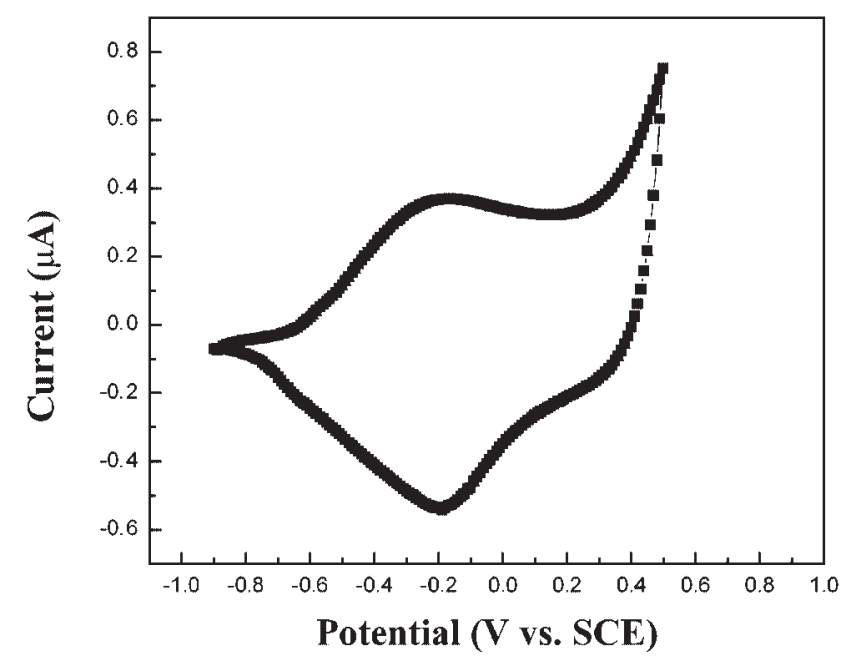

Figure 8. Cyclic voltammetry $(\mathrm{CV})$ of the conducting polymer-modified hydrogel. Within a single voltage sweep cycle, the anodic peak was at $-0.15 \mathrm{~V}$ and cathodic peak was $-0.19 \mathrm{~V}$ versus SCE.

factors or antiinflammatory drugs in the hydrogel layers.

The selectivity of each single electrode is an important feature for gathering the electric signals from single neurons. To ensure that the vertically grown conducting polymer did not significantly affect the adjacent electrode site, the impedance of the adjacent site was measured. Within experimental error, the measured impedance was equal to the unmodified hydrogel coating, and this implied that the cloud-like polypyrrole chains were not linked to the neighboring electrode. Presumably this will allow us to decrease the size of the electrode to get the more specific signals from single neurons without affecting charge transport efficiency, and should improve the ability to distinguish action potentials from different neurons. The value of the impedance of these probes at $1 \mathrm{kHz}$ was the lowest after $40 \mathrm{~min}$ of polymerization, with a value of $7 \mathrm{k} \Omega$. The dehydrated 40-min sample shows the impedance measured before reswelling in the buffer solution. The impedance of the dehydrated sample was a bit higher than that of the hydrated sample in the low frequency range $\left(<2 \times 10^{3} \mathrm{~Hz}\right)$. However, the impedance of the dehydrated sample was much higher than the hydrated one in the high frequency range $\left(>2 \times 10^{3} \mathrm{~Hz}\right)$. These differences reveal the important role of the local environment (density of the coating) in limiting charge transport to the probe. For polypyrrole incorporated with large counter ions such as PSS, the ionic movements are very different. The redox reaction is accompanied by the movement of small cations in and out of the films. Within a single voltage sweep cycle, the anodic peak was at $-0.15 \mathrm{~V}$ and cathodic peak was $-0.19 \mathrm{~V}$ versus SCE (Figure 8). The charge capacity of this novel conducting polymer 


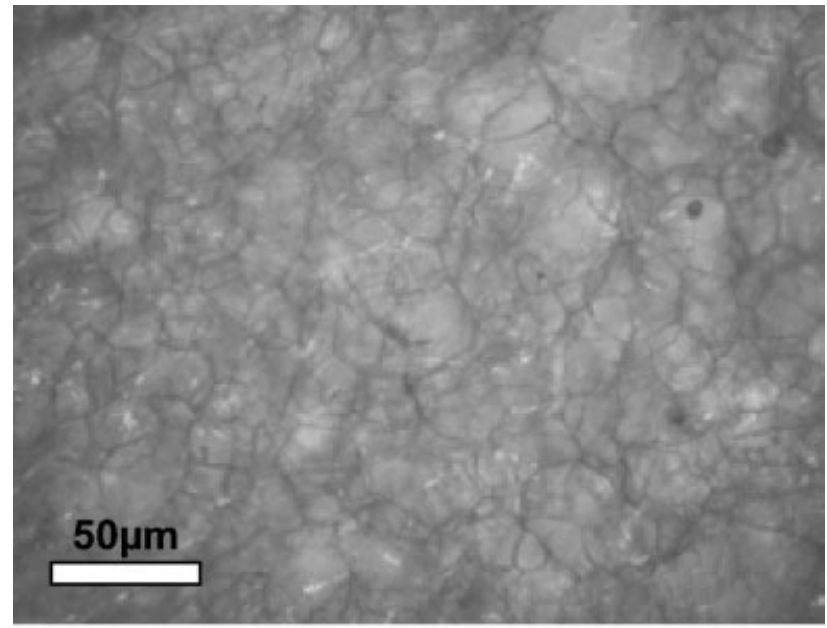

(a)

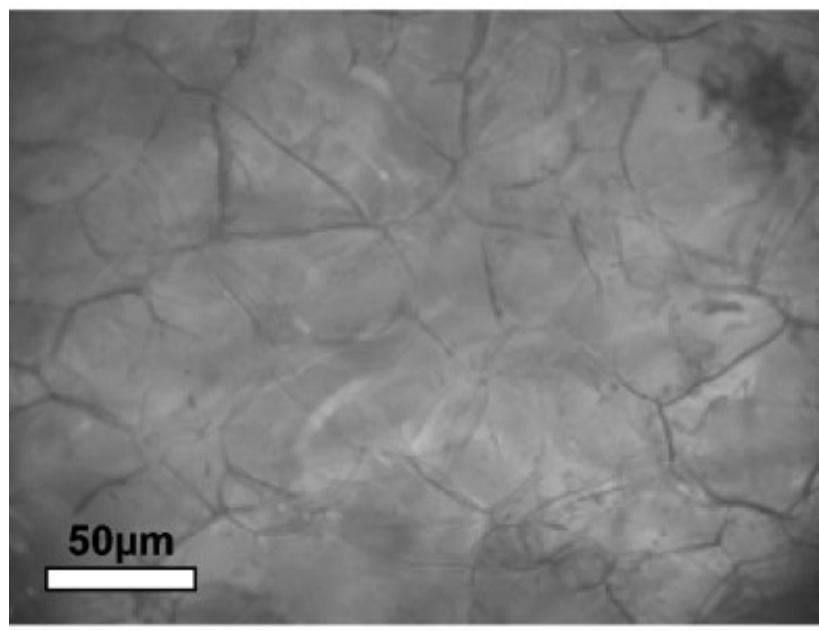

(b)

Figure 9. Optical microscope images of the hydrogel network: (a) liquid $\mathrm{N}_{2}$-frozen hydrogel, (b) freezer-frozen hydrogel.

in the hydrogel polymerized with the use of $5 \mu \mathrm{C}$ on a $1250 \mu \mathrm{m}^{2}$ was calculated as $7.01 \mu \mathrm{C}$. Normalizing with the use of the electrode area charge storage for this system gives $560 \mathrm{mC} / \mathrm{cm}^{2}$, which greatly exceeds the values of platinum $\left(6 \mathrm{mC} / \mathrm{cm}^{2}\right)^{29}$ and activated iridium $\left(61 \mathrm{mC} / \mathrm{cm}^{2}\right){ }^{30}$

To allow neovascularization and ingrowth of the neurons into the hydrogel layers, the pore size of the as-deposited MVG alginate hydrogel may not suffice. Lyophilized hydrogels that have a much larger pore size than that of intact hydrogels, and how these lyophilized hydrogels act as scaffolds for polymerization of conducting polymers, have also been investigated. The preparation and characterization of a three- dimensional porous hydrogel is recommended for creating a sufficient space to allow ingrowth of axons. This porous hydrogel is prepared with the use of a three-step procedure. ${ }^{27}$ The first step is gelation of the alginate with $\mathrm{CaCl}_{2}$, followed by freezing of the hydrogel and finally lyophilization to produce a porous hydrogel. In this study, liquid nitrogen $\left(-198^{\circ} \mathrm{C}\right)$ and a conventional refrigerator freezer $\left(-20^{\circ} \mathrm{C}\right)$ were used for solidification of the hydrogel. The lyophilized hydrogel at $-20^{\circ} \mathrm{C}$ shows a much more porous microstructure $(\sim 40-60 \mu \mathrm{m}$ in domain size) compared to the hydrogel dried at $-198^{\circ} \mathrm{C}(\sim 5-20-\mu \mathrm{m}$ domain size) as seen in the optical images (Figure 9), presumably due to the differences in the rate of solidification. The conducting polymer grown in the macroporous hydrogel scaffolds shows substantially different morphologies from that obtained in the homogeneous hy-

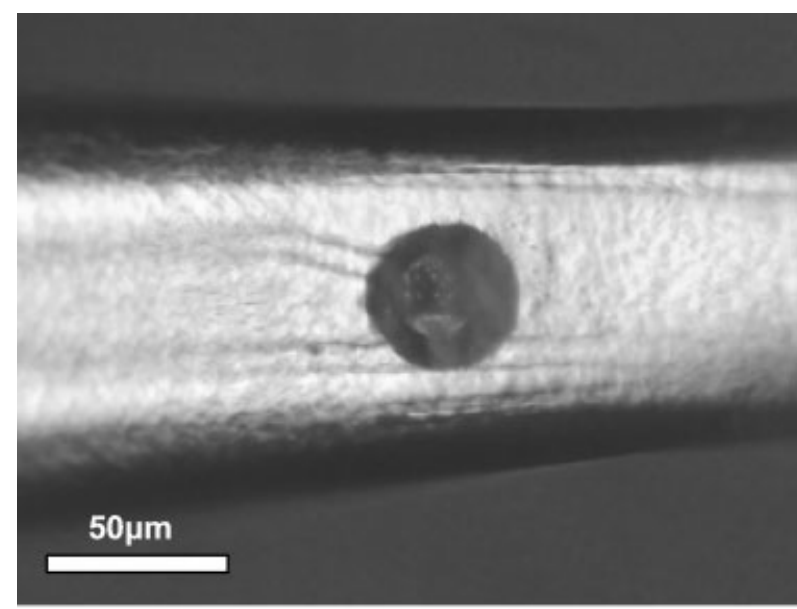

(a)

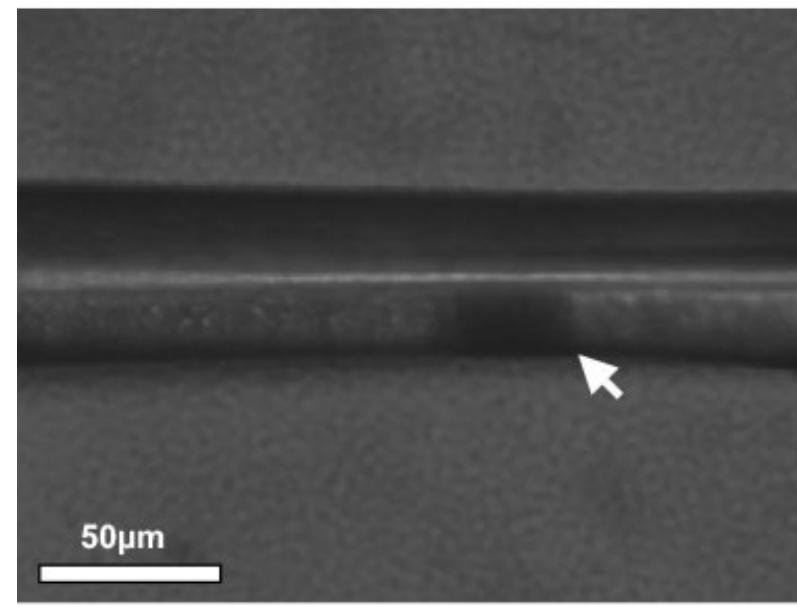

(b)

Figure 10. Optical microscope images of the extent of conducting polymer growth in the lyophilized hydrogel coating. (a) Top view and (b) lateral view of the conducting polymer. 


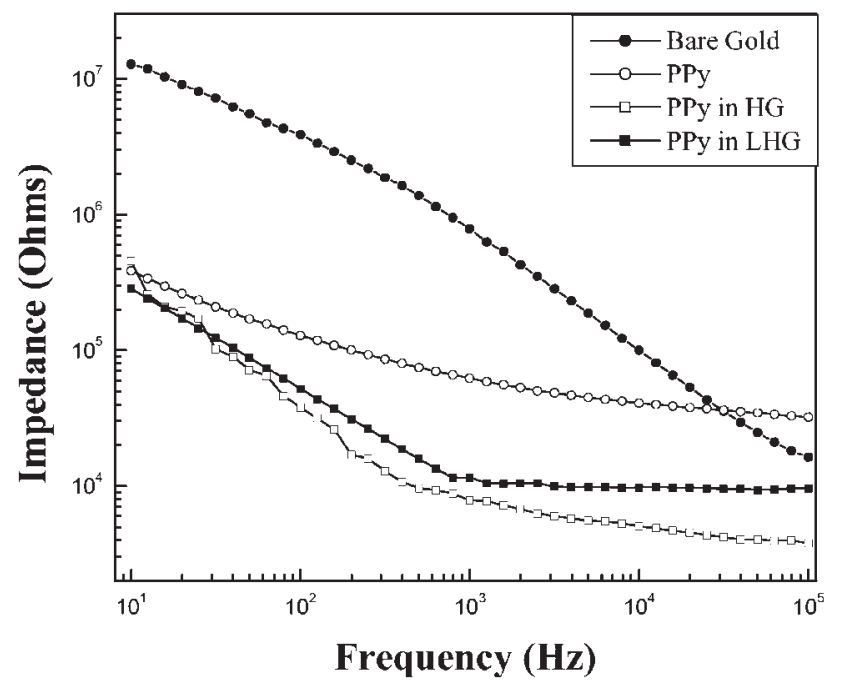

Figure 11. Impedance spectra as a function of frequency of bare gold electrode, film-like PPy, PPy grown in the intact hydrogel, and PPy in the lyophilized hydrogel.

drogel. Figure 10(a) shows the conducting polymer that was grown in the hydrogel frozen by liquid nitrogen. In contrast to the conducting polymer in the intact hydrogel [Figure 4(a)], the conducting polymer in the lyophilized hydrogel [Figure 10(b)] is more densely concentrated around the electrode. This is likely due to the larger pore size of the hydrogel allowing pyrrole monomer to more easily diffuse to the electrode during polymerization. There was not a large difference found between the morphology of the polypyrrole grown on the freezer-frozen hydrogel or the liquid $\mathrm{N}_{2}$-frozen hydrogel. This evidently means that the pore size of the hydrogel caused by either method of freezing is large enough to allow the pyrrole monomer to readily diffuse into the hydrogel. From the lateral view of these samples it is clear that the conducting polymer in the lyophilized hydrogel grows straighter toward the surface, instead of spreading out around the electrode, in contrast to the conducting polymer grown in the intact hydrogel [Figure 4(a)]. The value of the impedance of the PPy in lyophilized hydrogel (LHG) was slightly higher than that of PPy in intact hydrogel (HG) (Figure 11). The variation in the impedance of these systems was quite large, but always much lower than the direct deposition. This is consistent with the strong correlation of the impedance and the morphology of the PPy on the electrode. ${ }^{16,25}$

The impedance behavior at $1 \mathrm{kHz}$ as a function of deposition charge is shown in Figure 12. In the LHG, the impedance at $1 \mathrm{kHz}$ initially decreased sharply as the polymerization proceeded, then reached a minimum value at $3 \mu \mathrm{C}$. The impedance then gradually increased until $15 \mu \mathrm{C}$. Above $15 \mu \mathrm{C}$, the hydrogel was deformed because of the electric force in the monomer solution and then eventually slipped off the probe. In the $\mathrm{HG}$, the impedance decreased relatively slowly with charge. Unfortunately, after $15 \mu \mathrm{C}$ the impedance of the pyrrole in the hydrogel could not be measured because of the deformation of the hydrogel layers. Judging by the trend of this plot, the impedance of this system may be decreased more if the hydrogel could facilitate a longer polymerization time. Ongoing studies are investigating synthetic hydrogels and covalently cross-linked hydrogels as more stable scaffolds for the pyrrole polymerization.

\section{CONCLUSIONS}

By using a dipping method, hydrogels could be coated uniformly with a desired range of thicknesses along the shank of the micromachined probes to provide an additional mechanical buffer layer between the hard probe and the soft brain tissue, anchor the position of the probe in the tissue, and embed bioactive reagents. Pyrrole monomer could be reproducibly grown vertically through the hydrogel scaffold up to the surface without affecting the electronic properties of adjacent sites. This conducting polymer grown in the hydrogel scaffold has an extremely large amount of surface area and caused a significant reduction of the impedance of the electrode (more than two orders of magnitude). This should promote more efficient signal transport with neurons. The impedance of the electrode could be controlled by changing the polymerization time at fixed current density. The value of the impedance of these probes at $1 \mathrm{kHz}$ was the lowest

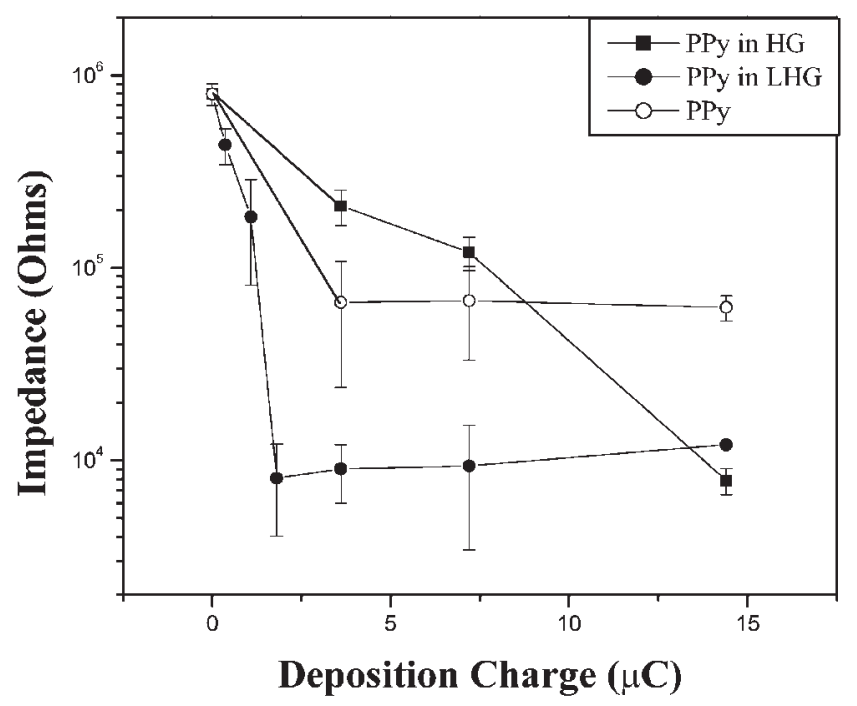

Figure 12. The impedance behavior of film-like PPy, PPy grown in the intact hydrogel (HG), and PPy in the lyophilized hydrogel (LHG) at $1 \mathrm{kHz}$ as a function of deposition charge. 
after 40 min of polymerization in the hydrogel scaffold, with a value of $7 \mathrm{k} \Omega$. This is much lower than that of polypyrrole film $(\sim 100 \mathrm{k} \Omega)$. The impedances of the pyrrole in the different types of LHG scaffolds were similar. The impedance behavior at $1 \mathrm{kHz}$ as a function of charge in the LHG showed the impedance sharply decreasing as the polymerization initiated, then reaching a minimum value at about $3 \mu \mathrm{C}$. For $\mathrm{HG}$, the impedance decreased relatively slowly with charge. Judging by the trend of decrease of impedance, presumably the impedance of this system may be decreased more if the hydrogel can facilitate longer polymerization times. Future work will involve synthetic hydrogels and covalently crosslinked hydrogels as scaffolds for the conducting polymers.

\section{References}

1. Buchko CJ. Processing and characterization of protein polymer thin films for surface modification of neural prosthetic devices [dissertation]. Ann Arbor: University of Michigan; 1997.

2. Athreya SA, Martin DC. Impedance spectroscopy of protein polymer modified silicon micromachined probes. Sensor Actuat A Phys 1999;72:203-216.

3. Niparko JK, Altschuler RA, Xue XL, Wiler JA, Anderson DJ. Surgical implantation and biocompatibility of central nervous system auditory prostheses. Ann Oto Rhinol Laryn 1989; 98:965-970.

4. Oweiss K, Wise M, Lopez C, Wiler JA, Anderson DJ. Chronic electrode-brain interface modeled with FEM. Adv Technol (Proceedings of the First Joint BMES/EMBS Conference Serving Humanity); 1999.

5. Rutten WLC. Selective electrical interfaces with the nervous system. Ann Rev Biomed Eng 2002;4:407-452.

6. Ratner B, Hoffman A, Schoen F, Lemons J. Biomaterial science: an introduction to materials in medicine. San Diego: Academic Press; 1996.

7. Mensinger AF, Anderson DJ, Buchko CJ, Johnson MA, Martin DC, Tresco PA, Silver RB, Highstein SM. Chronic recording of regenerating VIIIth nerve axons with a sieve electrode. J Neurophysiol 2000;83:611-615.

8. Ottenbrite RM, Huang SJ, Park K, Raphael M. Hydrogels and biodegradable polymers for bioapplications (ACS Symposium Series No. 627). Washington, DC: Oxford University Press; 1996.

9. Miller K, Chinzei K. Modeling of brain tissue mechanical properties: bi-phasic versus single-phase approach. Comput Methods Biomech Biomed Eng (Proceedings of the Third International Symposium); 1997.

10. Nyberg $\mathrm{T}$, Inganas $\mathrm{O}$, Jerregard $\mathrm{H}$. Polymer hydrogel microelectrodes for neural communication. Biomed Microdevices 2002;4:43-52.

11. Pernaut JM, Reynolds JR. Use of conducting electroactive polymers for drug delivery and sensing of bioactive molecules. A redox chemistry approach. J Phys Chem B 2000;104:4080-4090.

12. Wallace GG, Conductive electroactive polymers: Intelligent materials systems. Lancaster, PA: Technomic; 1997.
13. Attard GS, Bartlett PN, Coleman NRB, Elliott JM, Owen JR, Wang JH. Mesoporous platinum films from lyotropic liquid crystalline phases. Science 1997;278:838-840.

14. Sichel EK, Hoch R, Moy D, Tennent H. High power electrochemical capacitors based on carbon nanotube electrodes. Appl Phys Lett 1997;70:1480-1482.

15. Ghosh S, Inganas O. Conducting polymer hydrogels as 3D electrodes: applications for supercapacitors. Adv Mater 1999; 11:1214-1218

16. Cui X, Lee V, Raphael Y, Wiler J, Hetke J, Anderson DJ, Martin DC. Surface modification of neural recording electrodes with conducting polymer/biomolecule blends. J Biomed Mater Res 2001;56:261-272.

17. Schmidt CE, Shastri VR, Vacanti JP, Ranger R. Stimulation of neurite outgrowth using an electrically conducting polymer. Proc Natl Acad Sci USA 1997;94:8948-8953.

18. Foulds NC, Lowe CR. Enzyme entrapment in electrically conducting polymers-immobilization of glucose-oxidase in polypyrrole and its application in amperometric glucose sensors. J Chem Soc-Faraday Transac I 1986;82:1259-1264.

19. Wisniewski N, Moussy F, Reichert WM. Characterization of implantable biosensor membrane biofouling. Fresen J Anal Chem 2000;366:611-621.

20. Wang YB, Yuan RK, Yuan H, Chen ZH. Theoretical and experimental studies of conducting polymer polyaniline electrolyte interface by impedance spectroscopy. Synth Methods 1993;55: 1501-1508.

21. Hodgson AJ, John MJ, Campbell T, Georgevich A, Woodhouse S, Aoki T, Ogata N, Wallace GG. Integration of biocomponents with synthetic structures-use of conducting polymer polyelectrolyte composites. SPIE 1996;2716:164-176.

22. Garner B, Georgevich A, Hodgson AJ, Liu L. Wallace GG Polypyrrole-heparin composites as stimulus-responsive substrates for endothelial cell growth. J Biomed Mater Res 1999; 44:121-129.

23. Freemantle M. The endless polymer science frontier. Chem Eng News 2000;78:39-45.

24. Williams RL, Doherty PJ. A Preliminary assessment of poly(pyrrole) in nerve guide studies. J Mater Sci Mater Med. 1994; 5:429-433.

25. Cui X, Hetke J, Wiler J, Anderson DJ, Martin DC. Electrochemical deposition and characterization of conducting polymer polypyrrole/PSS on multichannel neural probes. Sensor Actuat A Phys 2001;93:8-18

26. Stein RB, Nichols TR, Jhamand AS, Davis L, Charles D. Stable long-term recordings from cat peripheral-nerves. Brain Res 1977;128:21-38

27. Shapiro L, Cohen S. Novel alginate sponges for cell culture and transplantation. Biomaterials 1997;18:583-590.

28. Rowley JA. Controlling myoblast phenotype with RGD-modified alginate matrices. [dissertation]. Ann Arbor: University of Michigan; 2001.

29. Stieglitz T, Beutel H, Schuettler M, Meyer J. Micromachined, polyimide-based devices for flexible neural interfaces. Biomed Microdevices 2000;2:283-294.

30. Robblee LS, Lefko JL, Brummer SB. Active IR-electrode suitable for reversible charge injection in saline solution. J Electrochem Soc 1983;130:731-733.

31. Kim BC, Spinks GM, Wallace GG Electroformation of conducting polymers in a hydrogel support matrix. Polymer 2000;41: 1783-1790. 